\title{
Variational Bayes and Mean Field Approximations for Markov Field Unsupervised Estimation
}

\section{Ali Mohammad-Djafari and Hacheme Ayasso}

\author{
Groupe Problèmes Inverses \\ Laboratoire des Signaux et Systèmes \\ UMR 8506 CNRS - SUPELEC - Univ Paris Sud 11 \\ Supélec, Plateau de Moulon, 91192 Gif-sur-Yvette, FRANCE.

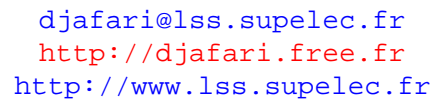

MLSP2009, 2-4 September 2009, Grenoble, France 


\section{Content}

- Gibbs-Markov fields and ML parameter estimation

- Variational energies and entropy

- Markov fields in imaging systems

- ML and MAP parameter estimation

- Variational Bayesian Approximation

- Mean Field Approximation

- Three classes of Markov fields in imaging systems

- Comparison of performances of VBA and MFA and MCMC

- Conclusions and Work in Progress

- Questions and Discussion 


\section{Gibbs-Markov fields and ML parameter estimation}

- Gibbs-Markov fields :

$$
p(x \mid \lambda)=\frac{1}{Z_{p}(\lambda)} \exp (-\lambda \mathcal{E}(x)),
$$

- Energy :

$$
\mathcal{E}(x)=\sum_{c \in \mathcal{C}} \Phi_{c}\left(x_{c}\right)
$$

$\mathcal{C}$ set of cliques, $\Phi_{c}($.$) potential$

- Partition function :

$$
Z_{p}(\lambda)=\int_{\mathcal{X}} \exp (-\lambda \mathcal{E}(x)) \mathrm{d} \boldsymbol{x}
$$

- Maximum likelihood (ML) estimation of $\lambda$ :

$$
\begin{gathered}
\widehat{\lambda}_{\mathrm{MV}}=\arg \max _{\lambda}\{\ln p(x \mid \lambda)\}=\arg \max _{\lambda}\{-\ln Z(\lambda)-\lambda \mathcal{E}(x)\} \\
\longrightarrow \quad \frac{-\partial \ln Z(\lambda)}{\partial \lambda}=\mathcal{E}(\boldsymbol{x})
\end{gathered}
$$




\section{Variational energies and entropy}

Main objective : Approximate $p$ by $q$ minimizing

- Kullback-Leibler divergence

$$
\mathrm{KL}(q \mid p)=<-\ln \frac{q}{p}>_{q}=-\int_{\mathcal{X}} q(x) \ln \frac{q(x)}{p(x)} \mathrm{d} x,
$$

- Variational free energy :

$$
F(q)=U(q)-H(q)
$$

- Variational average energy :

$$
U(q)=<\mathcal{E}(x)>_{q}=\int_{\mathcal{X}} q(x) \mathcal{E}(x) \mathrm{d} x
$$

- Variational entropy :

$$
H(q)=<-\ln q>_{q}=-\int_{\mathcal{X}} q(x) \ln q(x) \mathrm{d} x
$$




\section{Variational energies and entropy}

- Two main relations :

- Variational average energy :

$$
U(q)=-\ln Z(\lambda)+<\ln p>_{q}
$$

- Variational free energy :

$$
F(q)=-\ln Z(\lambda)+\mathrm{KL}(q \mid p)=F_{\text {Helmoltz }}+\mathrm{KL}(q \mid p) .
$$

- The main inequality:

- $F(q) \geq F_{\text {Helmoltz }}$, with equality when $q=p$.

- Main conclusion :

- Minimizing $F(q)$ is a good way to compute $F_{\text {Helmoltz }}=-\ln Z$ and use it where necessary. 


\section{Markov fields in imaging systems}

- $\boldsymbol{x}=\left\{x\left(\boldsymbol{r}_{i}\right), \boldsymbol{r}_{i} \in \mathcal{R}\right\}$ represent the pixels of an image $x(\boldsymbol{r})$

- $r_{i}$ spatial position of the pixel or voxel number $i$.

- Markov fields considered:

$$
\mathcal{E}(x)=\sum_{i} \sum_{j \in \mathcal{V} i} \Phi_{i}\left(x_{i}, x_{j}\right),
$$

where

$$
\Phi_{i}\left(x_{i}, x_{j}\right)=\Phi\left(x_{i}-x_{j}\right) \forall i
$$

- Expression :

$$
\begin{gathered}
p(x \mid \lambda)=\prod_{i} p\left(x_{i} \mid x_{j}, j \in \mathcal{V}(i)\right) \\
p\left(x_{i} \mid x_{j}, \in \mathcal{V}(i)\right) \propto \exp \left(-\lambda \sum_{j \in \mathcal{V}(i)} \Phi\left(x_{i}-x_{j}\right)\right)
\end{gathered}
$$




\section{ML and MAP parameter estimation}

- Maximum likelihood (ML) :

$$
\begin{aligned}
\widehat{\lambda}_{\mathrm{MV}} & =\arg \max _{\lambda}\{\ln p(x \mid \lambda)\} \\
& =\arg \max _{\lambda}\{-\ln Z(\lambda)-\lambda \mathcal{E}(\boldsymbol{x})\} \\
& \longrightarrow \quad \frac{-\partial \ln Z(\lambda)}{\partial \lambda}=\mathcal{E}(\boldsymbol{x})
\end{aligned}
$$

- Maximum A Posteriori (MAP) :

$$
\begin{aligned}
\widehat{\lambda}= & \arg \max _{\lambda}\{\ln p(\lambda \mid \boldsymbol{x})\} \\
= & \arg \max _{\lambda}\{-\ln Z(\lambda)-\lambda \mathcal{E}(\boldsymbol{x})+\ln \pi(\lambda)\} \\
& \longrightarrow \quad \frac{-\partial \ln (Z(\lambda) / \pi(\lambda))}{\partial \lambda}=\mathcal{E}(\boldsymbol{x})
\end{aligned}
$$


Three classes of Markov fields in imaging systems

- Generalized Gaussian (GG) :

$$
\mathcal{E}(\boldsymbol{x})=\sum_{i} \sum_{j \in \mathcal{V}(i)}\left|x_{i}-x_{j}\right|^{\beta}
$$

- Entropic (I-Distribution family) :

- First kind:

$$
\mathcal{E}(x)=\sum_{i} \sum_{j \in \mathcal{V}(i)} x_{j} \ln \frac{x_{j}}{x_{i}}-\left(x_{j}-x_{i}\right) .
$$

- Second kind:

$$
\mathcal{E}(x)=\sum_{i} \sum_{j \in \mathcal{V}(i)} x_{i} \ln \frac{x_{i}}{x_{j}}-\left(x_{i}-x_{j}\right) .
$$

- Potts and Ising models :

$$
\mathcal{E}(\boldsymbol{x})=-\sum_{i} \sum_{j \in \mathcal{V}(i)} \delta\left(x_{i}-x_{j}\right),
$$




\section{Variational Approximations Basics}

Main objective : Approximate $p$ by simpler $q \in \mathcal{Q}$ minimizing

$$
q(\boldsymbol{x})=\arg \min _{q \in \mathcal{Q}} K L(q: p) .
$$

and use it for all posterior computations.

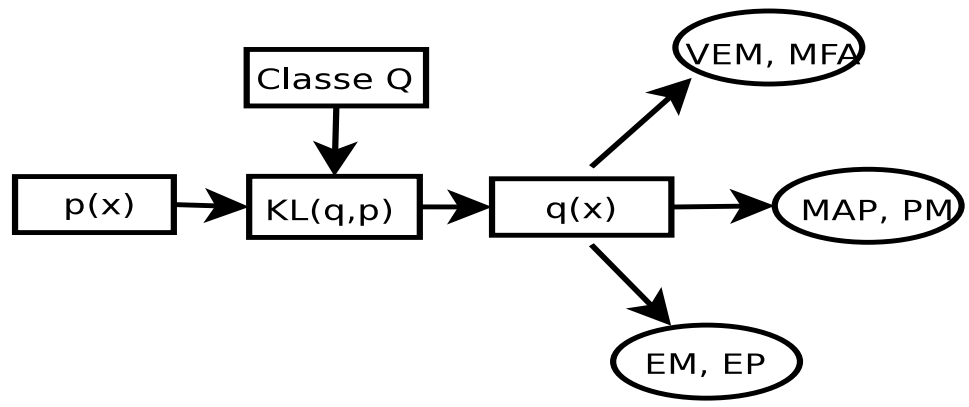

- No constraint $: \mathcal{Q}=\left\{q: \int q=1\right\} \longrightarrow q=p$

- Separable $: \mathcal{Q}=\left\{q: q(\boldsymbol{x})=\sum_{j} q_{j}\left(x_{j}\right)\right\} \longrightarrow$ VBA

- Parametric $: \mathcal{Q}=\left\{\boldsymbol{q}: q(\boldsymbol{x})=q_{\boldsymbol{\theta}}(\boldsymbol{x})\right\} \longrightarrow$ Parametric VBA

- Separable and parametric $\longrightarrow$ MFA 


\section{Variational Bayesian Approximation}

- Main objective : Approximate $p$ by a separable

$$
q \in \mathcal{Q}=\left\{q: q(x)=\sum_{j} q_{j}\left(x_{j}\right)\right\}
$$

minimizing $\mathrm{KL}(q: p)$.

- The solution has the form :

$$
q_{i}\left(x_{i}\right)=\frac{1}{Z_{i}(\lambda)} \exp \left(-\lambda\langle\mathcal{E}(x)\rangle_{\prod_{j \neq i} q_{j}}\right)
$$

- Iterative

- Needs the computation of $\langle\mathcal{E}(x)\rangle_{\prod_{j \neq i} q_{j}}$

- Analytic expression if $p(x)$ is in Exponential family

- In our application only parametric form is usable. 


\section{Mean Field Approximation}

Example :

$$
p(x \mid \lambda)=\prod_{i} p\left(x_{i} \mid x_{j}, j \in \mathcal{V}(i)\right) \text { with }
$$

$$
p\left(x_{i} \mid x_{j}, j \in \mathcal{V}(i)\right)=\frac{1}{Z_{i}(\lambda)} \exp \left(-\lambda \sum_{j \in \mathcal{V}(i)} \Phi\left(x_{i}-x_{j}\right)\right),
$$

is approximated by

$q(\boldsymbol{x} \mid \lambda)=\prod_{i} q\left(x_{i} \mid \bar{x}_{j}, j \in \mathcal{V}(i)\right)$ with

$$
q\left(x_{i} \mid \bar{x}_{j}, j \in \mathcal{V}(i)\right)=\frac{1}{Z_{i}(\lambda)} \exp \left(-\lambda \sum_{j \in \mathcal{V}(i)} \Phi\left(x_{i}-\bar{x}_{j}\right)\right),
$$

$\bar{x}_{j}$ becomes a parameter to be estimated such that $\mathrm{KL}(q: p)$ be minimized. 


\section{VBA $=$ MFA for Gaussian, Entropic, Potts and Ising}

- Gaussian Case

$\ln \left(q_{i}\left(x_{i}\right)\right) \propto-\frac{\lambda}{2} \sum_{j \in \mathcal{V}(i)}\left[x_{i}^{2}-2 x_{i} \tilde{\mu}_{j}+\tilde{\mu}_{j}^{2}+\tilde{v}_{j}\right] \Rightarrow q_{i}\left(x_{i}\right)=\mathcal{N}\left(\tilde{\mu}_{i}, \tilde{v}_{i}\right)$

with

$$
\tilde{\mu}_{i}=\frac{1}{|\mathcal{V}|} \sum_{j \in \mathcal{V}(i)} \tilde{\mu}_{j} \text { and } \tilde{v}_{i}=\frac{1}{|\mathcal{V}| \lambda}
$$

Bayesian estimation of $\lambda: \pi(\lambda)=\Gamma(a, b) \longrightarrow p(\lambda \mid x)=\Gamma(\hat{a}, \hat{b})$ where $\hat{a}=\left[\frac{1}{a}+\sum_{i \in \mathcal{R}}\left(x_{i}-\tilde{\mu}_{i}\right)^{2}\right]^{-1}$ and $\hat{b}=\frac{|\mathcal{R}|}{2}+b$. 


\section{VBA $=$ MFA for Gaussian, Entropic, Potts and Ising}

- Entropic Case 1

$$
\begin{gathered}
\left.\ln \left(q\left(x_{i}\right)\right) \propto-\lambda \sum_{j \in \mathcal{V}(i)} \exp \left[x_{j}\right]\right]_{j} \ln \frac{\exp \left[x_{j}\right]_{q_{j}}}{x_{i}}-x_{i}+\exp \left[x_{j}\right]_{q_{j}} \\
Z_{i}(\lambda)=e^{-\lambda \tilde{\mu}_{i} \ln \tilde{\mu}_{i}+\lambda \tilde{\mu}_{i}} \lambda^{-\lambda \tilde{\mu}_{i}-1} \Gamma\left(\lambda \tilde{\mu}_{i}+1\right)
\end{gathered}
$$

with $\tilde{\mu}_{i}=\sum_{j \in \mathcal{V}(i)} \exp \left[x_{j}\right]_{q_{j}}$.

- Entropic case 2

$$
\begin{gathered}
\ln \left(q\left(x_{i}\right)\right) \propto-\lambda \sum_{j \in \mathcal{V}(i)} x_{i} \ln \frac{x_{i}}{e^{-\exp \left[\ln x_{j}\right] q_{j}}}+x_{i}-\exp \left[x_{j}\right] q_{j} \\
Z_{i}(\lambda)=\frac{e^{-\lambda \tilde{\mu}_{i}}}{\lambda} \alpha\left(\lambda \tilde{\mu}_{i}\right)
\end{gathered}
$$

with :

$\tilde{\mu}_{i}=e^{-\sum_{j \in \mathcal{V}(i)} \exp \left[\ln x_{j}\right] a_{j}}$ and $\alpha(a)=\int_{0}^{\infty} a^{y} e^{-y \ln y+y} d y$. 


\section{VBA $=$ MFA for Gaussian, Entropic, Potts and Ising}

- Ising and Potts

$$
q_{i}\left(x_{i}=k\right)=\frac{\exp \left(-\lambda \sum_{j \in \mathcal{V}(i)} q_{j}\left(x_{j}=k\right)\right)}{\sum_{k=1}^{K} \mathrm{e}^{-\lambda \sum_{j \in \mathcal{V}(i)} q_{j}\left(x_{j}=k\right)}}
$$

$x_{j} \in\{1, \ldots, K\}$ is the general case and $x_{j} \in\{0,1\}$ is the Ising model case. 
Simulation results : Comparison between VBA, MFA and MCMC 


\section{Simulations}
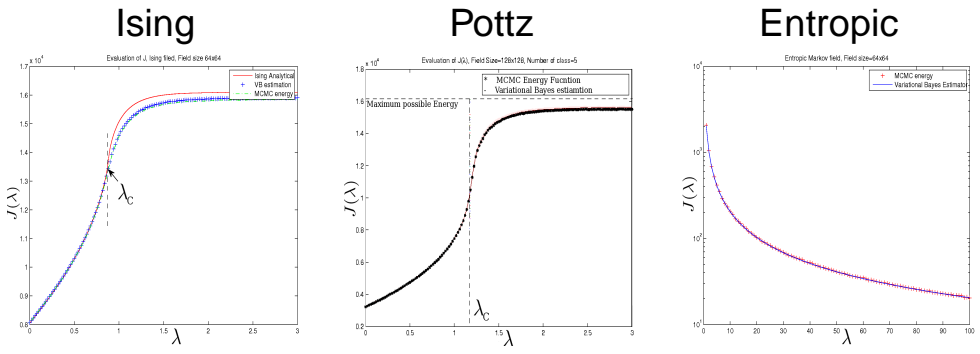

a

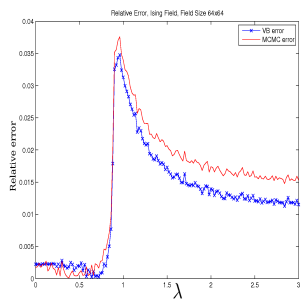

C

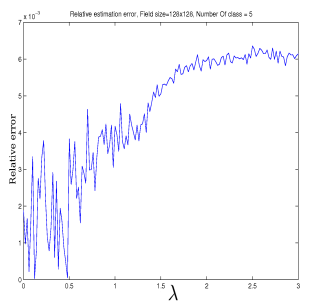

b

d

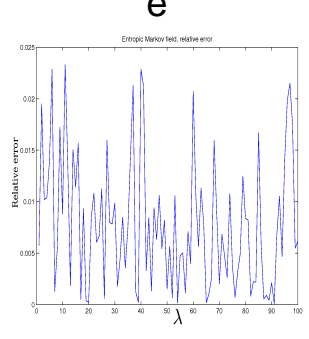

f 


\section{Simulations}

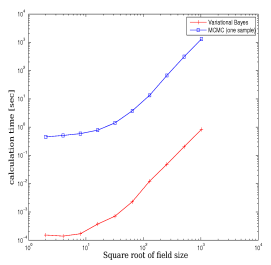

a

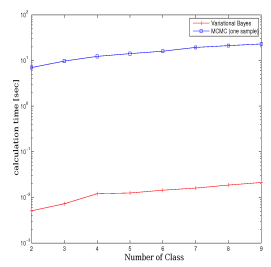

b 


\section{Conclusions}

- Gauss-Markov-Potts are useful prior models for images incorporating regions and contours

- Bayesian computation needs often pproximations (Laplace, MCMC, Variational Bayes)

- Application in different CT systems (X ray, Ultrasound, Microwave, PET, SPECT) as well as other inverse problems

Work in Progress and Perspectives :

- Efficient implementation in 2D and 3D cases using GPU

- Evaluation of performances and comparison with MCMC methods

- Application to other linear and non linear inverse problems :

(PET, SPECT or ultrasound and microwave imaging) 